\title{
Opioid growth factor improves clinical benefit and survival in patients with advanced pancreatic
} cancer

\author{
Jill P Smith' \\ Sandra I Bingaman' \\ David T Mauger ${ }^{2}$ \\ Harold H Harvey' \\ Laurence M Demers ${ }^{3}$ \\ Ian S Zagon ${ }^{4}$ \\ 'Departments of Medicine, ${ }^{2}$ Public \\ Health Sciences, ${ }^{3}$ Pathology, and \\ ${ }^{4}$ Neurosciences and Anatomy, \\ Pennsylvania State University, College \\ of Medicine, Hershey Medical Center, \\ Hershey, PA, USA
}

This article was published in the following Dove Press journal:

Open Access Journal of Clinical Trials

25 March 2010

Number of times this article has been viewed
Background: Advanced pancreatic cancer carries the poorest prognosis of all gastrointestinal malignancies. Once the tumor has spread beyond the margins of the pancreas, chemotherapy is the major treatment modality offered to patients; however, chemotherapy does not significantly improve survival.

Objective: Opioid growth factor (OGF; [Met ${ }^{5}$-enkephalin) is a natural peptide that has been shown to inhibit growth of pancreatic cancer in cell culture and in nude mice. The purpose of this study was to evaluate the effects of OGF biotherapy on subjects with advanced pancreatic cancer who failed chemotherapy.

Methods: In a prospective phase II open-labeled clinical trial, 24 subjects who failed standard chemotherapy for advanced pancreatic cancer were treated weekly with OGF $250 \mu \mathrm{g} / \mathrm{kg}$ intravenously. Outcomes measured included clinical benefit, tumor response by radiographic imaging, quality of life, and survival.

Results: Clinical benefit response was experienced by 53\% of OGF-treated patients compared to historical controls of $23.8 \%$ and $4.8 \%$ for gemcitabine and 5-fluorouracil (5-FU), respectively. Of the subjects surviving more than eight weeks, $62 \%$ showed either a decrease or stabilization in tumor size by computed tomography. The median survival time for OGF-treated patients was three times that of untreated patients ( 65.5 versus 21 days, $p<0.001)$. No adverse effects on hematologic or chemistry parameters were noted, and quality of life surveys suggested improvement with OGF.

Limitations: Measurements other than survival were not allowed in control patients, and clinical benefit comparisons were made to historical controls.

Conclusion: OGF biotherapy improves the clinical benefit and prolongs survival in patients with pancreatic cancer by stabilizing disease or slowing progression. The effects of OGF did not adversely alter patient quality of life. The use of OGF biotherapy at earlier stages of disease or in combination with other chemotherapeutic agents may further improve the outcome of this malignancy.

Keywords: phase II, pancreatic cancer, biotherapy, quality of life

\section{Introduction}

Pancreatic cancer is a fatal malignancy estimated to occur in approximately 38,000 Americans in 2009 and ranks as the fourth most common cause of cancer-related mortality in the United States. ${ }^{1}$ With a five-year survival rate of less than $1 \%$, pancreatic cancer remains the lowest of all malignancies, and the median survival is three to six months with only $10 \%$ of patients surviving two years. ${ }^{2}$ Surgical resection of the tumor improves median survival to 17-20 months, but five-year survival is still less than $10 \% .^{3}$ 
Pancreatic cancer is most commonly diagnosed in advanced stages, and therefore, chemotherapy remains the major treatment modality for this disease. ${ }^{4}$ Among the chemotherapeutic agents tested, gemcitabine has been used the most either alone or in combination with other agents..$^{5-7}$ Although various combinations of other drugs with gemcitabine ${ }^{5}$ or with concurrent radiation ${ }^{8}$ appear to improve the response rates, a clear survival benefit has not yet been demonstrated. Because of the overall poor response to chemotherapeutic agents, researchers have been evaluating novel approaches to treat pancreatic cancer by targeting specific markers. ${ }^{9}$

When pancreatic cancer is diagnosed in the advanced state and is not resectable, much of the medical management focuses on palliation of tumor-related symptoms, ie, pain, functional impairment and weight loss. Burris and colleagues studied the effects of chemotherapy on clinical benefit assessment in which these three tumor-related parameters were measured. ${ }^{10}$ For subjects to achieve a positive overall clinical benefit, they must demonstrate improvement in at least one parameter (pain, performance status, or weight) without having a decline in any of the others, and this benefit had to be sustained for a minimum of four weeks. ${ }^{10}$ With the short survival reported with pancreatic cancer, researchers have focused on other potential advantages of drug regimens such as the quality of life, drug side effects, and overall well-being.

Growth factors, molecules that transmit stimulatory/ inhibitory signals between cells, and growth factor receptors represents an area of heightened interest in understanding the pathophysiology, diagnosis, and treatment of pancreatic cancer. ${ }^{11-13}$ One group of peptides, the endogenous opioids, is important in neoplasia, including pancreatic cancer, as well as homeostasis, cellular renewal, wound healing, and angiogenesis. ${ }^{14}$ The endogenous opioid, $\left[\mathrm{Met}^{5}\right]$-enkephalin (termed opioid growth factor [OGF]) is a constitutively expressed pentapeptide that interacts with the OGF receptor (OGFr) to inhibit pancreatic cancer growth both in vitro ${ }^{15,16}$ and in vivo. ${ }^{17}$ The action of OGF is targeted to DNA synthesis, ${ }^{16}$ and is directed toward the $\mathrm{G}_{1} / \mathrm{S}$ interface of the cell cycle. ${ }^{18}$ Pharmacological, biochemical, and physiological analysis shows that OGFr has a unique set of characteristics that distinguishes it from classical opioid receptors (eg, $\mu, \delta$, and $\kappa$ ) in that its location is on the nuclear membrane and its function involves growth. ${ }^{14-16}$ Immunoelectron microscopy studies reveal that OGFr is located on the outer nuclear envelope, forms a complex with OGF, translocates through the nuclear pore, and localizes to the heterochromatin. ${ }^{19}$ OGFr has been cloned and sequenced in humans ${ }^{16}$ where the cDNA $(2.4 \mathrm{~kb})$ encodes a protein of 677 amino acids and the chromosomal location of OGFr is $20 \mathrm{q} 13.3 .{ }^{20}$

The safety and toxicity of exogenously administered OGF has been determined in humans, and the maximum tolerated dose has been reported in a phase I clinical trial. ${ }^{21}$ All of the subjects in the phase I trial had advanced pancreatic cancer and were not candidates for surgical resection. One-half had not received any prior chemotherapy. The average survival in subjects treated chronically with OGF in this study was 9.1 months. ${ }^{21}$ The purpose of the current study was to evaluate the clinical benefit of OGF on cancer-associated signs and symptoms, tumor parameters, survival, as well as quality of life.

\section{Patients and methods \\ Patient eligibility and selection}

Adult patients, aged 18 years or older, with histologically confirmed and advanced (stage 3 or 4) unresectable pancreatic adenocarcinoma, who had failed chemotherapy were eligible for the study. A Karnofsky performance status ${ }^{22}$ of $50 \%$ or greater was required. Exclusion criteria for the study are listed in Table 1. Patients who elected no further treatment for their disease and were admitted to hospice care served as the control group.

At the screening visit, patients read and signed the informed consent, the Karnofsky score was determined, and disease stage assessed. An abdominal computed tomography (CT) scan was performed to determine the baseline tumor marker size.

The protocol was approved by the Institutional Review Board (IRB) of the Pennsylvania State University, College of Medicine and the US Food and Drug Administration (FDA; IND\# 50,987).

\section{Treatment regimen}

The investigational drug, OGF, chemically is the five amino acid pentapeptide, $\left[\mathrm{Met}^{5}\right]$-enkephalin. The compound was synthesized and HPLC purified by Bachem (Carlsbad, CA) according to good manufacturing practice standards, and vialed as a $10 \mathrm{mg}$ lyophilized powder. Samples were tested for sterility (endotoxin, fungus, and bacterial) by Celsis Laboratory Group Laboratories (St. Louis, MO). OGF ( $250 \mu \mathrm{g} / \mathrm{kg}$ ) was administered intravenously in a volume of $50 \mathrm{~mL}$ of sterile saline over 45 minutes each week. Treatment was initiated within 10 days of the screening visit. Weight was recorded weekly and baseline vital signs were recorded prior to each infusion and at 15, 30, and 45 minutes during the infusion 
Table I Exclusion criteria

\begin{tabular}{ll}
\hline Medical conditions & Laboratory values \\
\hline - History of another cancer (excluding basal cell & Leukocyte count $<3,500 / \mathrm{mm}^{3}$ \\
skin cancer or stage I cervical cancer) & Absolute neutrophil count $<1,500 / \mathrm{mm}^{3}$ \\
- Uncontrolled cardiovascular disease & Hemoglobin $<8.5 \mathrm{~g} / \mathrm{dL}$ \\
- Myocardial infarction in prior six months & Blood urea nitrogen $>30 \mathrm{mg} / \mathrm{dL}$ \\
- Poorly controlled asthma, COPD, & Creatinine $>2.0 \mathrm{mg} / \mathrm{dL}$ \\
$\quad$ diabetes, or seizures & Total bilirubin $>4.0 \mathrm{mg} / \mathrm{dL}$ \\
- Hepatic failure or renal failure & Platelet count $<100,000 / \mathrm{mm}^{3}$ \\
- Known brain metastases & $\mathrm{INR}>1.8$ (unless on warfarin) \\
- Pregnant or nursing females & Sodium $<130 \mathrm{mmol} / \mathrm{L}$ \\
- Serious infections requiring antibiotics two & Potassium $<3.2 \mathrm{mmol} / \mathrm{L}$ \\
$\quad$ weeks prior to study & Glucose $>300$ or $<60 \mathrm{mg} / \mathrm{dL}$ \\
\hline
\end{tabular}

Abbreviations: COPD, chronic obstructive pulmonary disease; INR, international normalized ratio.

and 30 minutes post-infusion. Quality of life surveys were completed at baseline and every four weeks thereafter; these included: the Sickness Impact Profile, ${ }^{23}$ the Beck Depression Inventory, ${ }^{24}$ and the McGill Pain Questionnaire. ${ }^{25}$

Every four weeks, patients underwent a history and physical examination, and completed quality of life surveys, and a monthly pain diary. Monthly laboratory tests included the following: complete blood count, differential, platelet count, liver profile, renal profile, electrolytes, calcium, prothrombin time, CA19-9, and [Met] $]^{5}$-enkephalin blood levels ) to rule out any hematologic toxicity and to make appropriate adjustments in drug dosage if necessary according to the World Health Organization (WHO) criteria. ${ }^{26}$ Moreover, a urine pregnancy test was performed on women of child bearing potential.

\section{Parameters of measurement}

Clinical benefit was a principal outcome of the study which evaluated three common debilitating signs and symptoms found in most patients with advanced pancreatic cancer including pain, functional status, and weight loss according to previously published criteria in pancreatic cancer patients. ${ }^{10}$ Pain intensity was scored on a scale of $0-100$ and patients kept a diary for analgesic consumption. A positive score was improvement of pain intensity or a decrease in analgesic consumption of $\geq 50 \%$ from baseline and sustained for $\geq 4$ weeks; a negative score was any worsening; and a stable score was anything else. Performance status was determined by the Karnofsky performance scoring system ${ }^{22}$ with a positive score being an improvement of $\geq 20$ points from baseline sustained for $\geq 4$ weeks; a negative score was any worsening of $\geq 20$ points; and a stable score was any other result. Weight was measured weekly at each visit and a positive score was a weight gain (without fluid retention) of $\geq 7 \%$ from baseline and sustained for $\geq 4$ weeks and a nonpositive weight score was any other result. Clinical benefit was compared to historical controls using gemcitabine and 5-Fluorouracil (5-FU) to treat advanced pancreatic cancer.

The efficacy of OGF on tumor burden was determined by monthly plasma tumor marker levels (CA19-9) and radiographic CT imaging every eight weeks. A response was defined according to the RECIST (Response Evaluation Criteria In Solid Tumors) criteria. ${ }^{27}$ Patients with progression of disease were terminated from the study but these patients were followed until death.

Survival was another end point of this study for both the OGF-treated and untreated hospice control subjects. Survival was determined from the first day of enrollment into the study until death for OGF-treated subjects, and from the date of enrollment into hospice care until death for control subjects. The Kaplan-Meier analysis was performed to determine the patient survival over time comparing OGF-treated to hospice-referred subjects. Survival tables were generated to determine the cumulative proportion of subjects surviving at specific time points in each group.

Another outcome parameter assessed in this study was the quality of life using the three questionnaires mentioned earlier to assess quality of life, depression, and pain. Safety and toxicity was monitored and graded according to the common terminology criteria for adverse events (version 3.0). ${ }^{28}$ Pharmacokinetic studies were assessed by measuring $\left[\mathrm{Met}^{5}\right]$-enkephalin plasma levels with an iodinated radioimmunoassay (RIA) using reagents obtained from Peninsula Laboratories (San Carlos, CA). 


\section{Statistics}

Clinical benefit was evaluated using Chi-square analysis comparing positive responders to those from historical controls who were treated with either gemcitabine or 5-FU chemotherapy. The Kaplan-Meier method was used to estimate the survival curve for patients on OGF compared to no treatment by examining the $95 \%$ confidence interval for median survival of patients. ${ }^{29}$ Kaplan-Meier analysis was performed using PROC LIFETEST. Measure of response was analyzed by ordinal logistic regression (using PROC LOGISTIC). Mean laboratory values for each subject were calculated and all statistical analyses were carried out using the SAS statistical software system (version 8.1; SAS Institute, Cary, $\mathrm{NC}$ ). The sample size was determined based upon the safety and survival information of OGF treated patients in the phase I clinical trial. ${ }^{21}$ With an estimated survival of OGF subjects of 9.1 months compared to historical controls for 5-FU and gemcitabine of 4.4 and 5.6 months, ${ }^{30}$ respectively, and assuming at least $80 \%$ power 25 subjects were estimated for this study. It was assumed that the clinical benefit of OGF would be approximately twice that of gemcitabine since survival was twice that of the historical control.

Survival data were calculated between the OGF-treated patients and those who enrolled into hospice care. Statistical analysis was performed on values obtained up through week 8 . Since the number of surviving subjects beyond week 8 decreased, statistical analysis was not performed due to the low numbers. Mean laboratory blood values were determined and analyzed by analysis of variance for statistical differences between baseline, week 4, and week 8 of OGF administration.

Descriptive statistics were calculated for each of the outcome measures described above at the baseline visit and at weeks 4 and 8 . The paired t-test was used to assess differences in outcome measures between week 4 and baseline, and between week 8 and baseline. Differences in outcome measures between baseline and later visits were not formally assessed due to insufficient numbers of subjects.

\section{Results}

\section{Patient characteristics}

Twenty-four adults with advanced nonresectable pancreatic adenocarcinoma who had failed standard chemotherapy for their disease were enrolled in the study. Demographics and prior treatment of study subjects is shown in Table 2 . Almost all of the OGF-treated patients (96\%) had failed standard care with gemcitabine. Eight subjects received gemcitabine as their only chemotherapeutic agent whereas all
Table 2 Patient baseline characteristics $(\mathrm{N}=24)$

\begin{tabular}{|c|c|c|}
\hline Characteristic & No & $\%$ \\
\hline \multicolumn{3}{|l|}{ Age, years } \\
\hline$($ mean \pm SEM) & & $61.6 \pm 2.3$ \\
\hline Range & & $46-80$ \\
\hline \multicolumn{3}{|l|}{ Gender } \\
\hline Male & 12 & 50 \\
\hline Female & 12 & 50 \\
\hline \multicolumn{3}{|l|}{ Stage of disease } \\
\hline III & 1 & $4 \%$ \\
\hline IV & 23 & $96 \%$ \\
\hline \multicolumn{3}{|l|}{ Karnofsky status } \\
\hline$($ mean \pm SEM) & & $79.2 \pm 2.7$ \\
\hline Range & & $50-100$ \\
\hline \multicolumn{3}{|l|}{ Pain intensity score (mm) } \\
\hline$($ mean \pm SEM) & & $43 \pm 5.0$ \\
\hline Range & & $20-100$ \\
\hline \multicolumn{3}{|l|}{ Prior therapy } \\
\hline Gemcitabine & 23 & 96 \\
\hline 5-Fluorouracil & 4 & 16 \\
\hline Cisplatin & 2 & 8 \\
\hline Oxaliplatin & 4 & 16 \\
\hline Capecitabine & 5 & 20 \\
\hline Tarceva & 3 & 12 \\
\hline Irinotecan & 2 & 8 \\
\hline Investigational & 4 & 16 \\
\hline Prior surgery with recurrence & 4 & 16 \\
\hline Chemosensitizing radiation therapy & 5 & 20 \\
\hline
\end{tabular}

Abbreviation: SEM, standard error of mean.

of the remaining patients had failed multiple drug regimens subsequent to gemcitabine. Radiation therapy was used in six patients along with either 5-FU or gemcitabine as the chemosensitizer. One patient had stage 3 disease but was not considered a surgical candidate due to encasement of tumor around the blood vessels and the size of tumor. Twenty-three of the 24 subjects had metastatic disease to liver, peritoneum, lungs, skin, and/or peripheral lymph nodes.

The control group for survival analysis included a similar cohort of 166 subjects who met the same inclusion criteria but opted not to have further treatment and were discharged to hospice care. Their mean age was the same as those treated with OGF but other baseline measurements were not permitted.

\section{Clinical outcome}

Fifteen subjects were evaluable for at least eight weeks to determine clinical benefit (since any change had to be 
sustained for at least four weeks). Seven of the 15 subjects $(47 \%)$ had a positive result for pain intensity and narcotic analgesic consumption (Figure 1A). Seven of 15 subjects (47\%) also had improved or stable Karnofsky performance status that had a positive score for pain (Figure 1B). One subject who was categorized as "stable" in the primary measures categories A (pain) and B (Karnofsky performance status) had improved appetite, food consumption and weight gain. Therefore, this subject was added to the other seven with positive primary measures from A and B making a total of eight subjects (53\%) with an overall clinical benefit from OGF therapy (Figure 1C). The clinical benefit was twice that compared to historical controls treated with gemcitabine $(23.8 \%)$ and 10 -fold better than those receiving 5 -FU $(4.8 \%)^{10}$ (significantly greater than 5-FU, $p<0.002$ ).

Thirteen subjects receiving OGF treatment were evaluated by radiographic imaging at eight weeks and, of these
$62 \%$ showed significant reduction in tumor size (partial response $[\mathrm{PR}]=$ three subjects) or stabilization of disease (stable disease $[\mathrm{SD}]=$ five subjects). Serial CT scans of the abdomen in a patient with stable disease until week 24 are shown in Figure 2.

CA19-9 levels were marked elevated (14,079 $\pm 7,212 \mathrm{U} / \mathrm{mL})$ upon enrollment into the study reflecting the massive tumor burden in the study subjects. Although the values increased at week-4 $(25,108 \pm 18,116 \mathrm{U} / \mathrm{mL} ; p=0.02)$. These values decreased again at week $8(14,614 \pm 9,642 \mathrm{U} / \mathrm{mL} ; p=0.05)$ not as a result of those subjects with high CA19-9 values dying, but this change corresponded to decreasing tumor size. Of the subjects who were evaluable beyond the 8-week follow-up $(\mathrm{N}=13)$, five had a transient decrease in the CA19-9 levels (but all increased again by week 12), one remained unchanged, and seven showed increased values.

The median survival time for OGF-treated patients was 65.5 days (mean survival of $115 \pm 23.1$ days) and

\section{Pain intensity}

\begin{tabular}{|c|c|c|c|c|}
\hline A. Pain parameters & & + & Stable & - \\
\hline \multirow{3}{*}{$\begin{array}{l}\text { Analgesic } \\
\text { consumption }\end{array}$} & + & 0 & 0 & 1 \\
\hline & Stable & 7 & 3 & 1 \\
\hline & - & 1 & 2 & 0 \\
\hline
\end{tabular}

Total positive $7=47 \%$

Karnofsky performance status

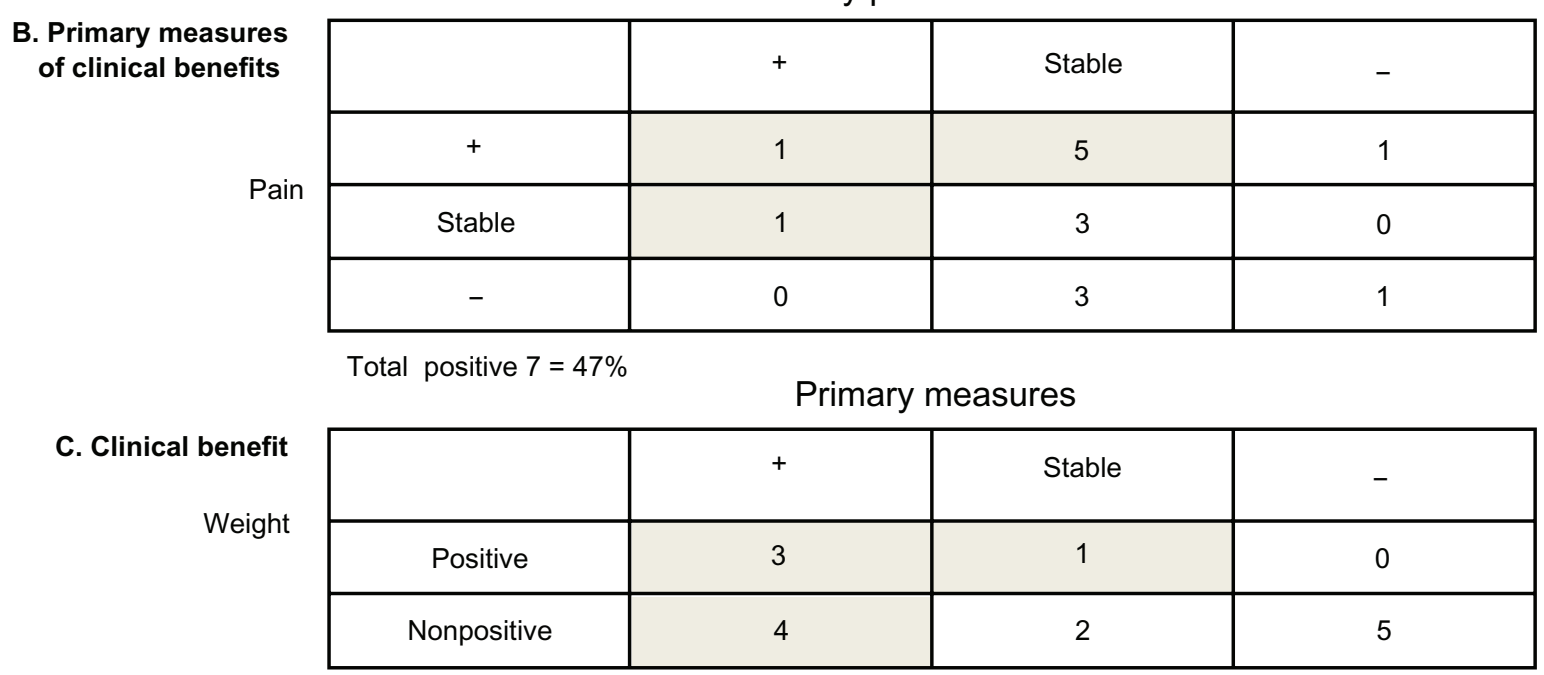

Total positive $8=53 \%$

Figure I Clinical benefit of opioid growth factor (OGF) in advanced pancreatic cancer. Clinical benefit as determined by improvement or stability in three cancer-related signs and symptoms (pain, functional status, and weight loss) were determined in OGF-treated subjects. A) Pain measures of clinical benefit based upon pain intensity and narcotic analgesic consumption B) Primary measures of clinical benefit based on Karnofsky performance status and pain. C) Forty-seven percent had a positive clinical benefit that was sustained a minimum of eight weeks with OGF treatment for pain and performance status but the overall clinical benefit was $53 \%$ utilizing both the primary outcomes (pain and performance status) and the secondary outcomes (weight gain). The Total positive was determined by the sum of the three shaded boxes in each group. 

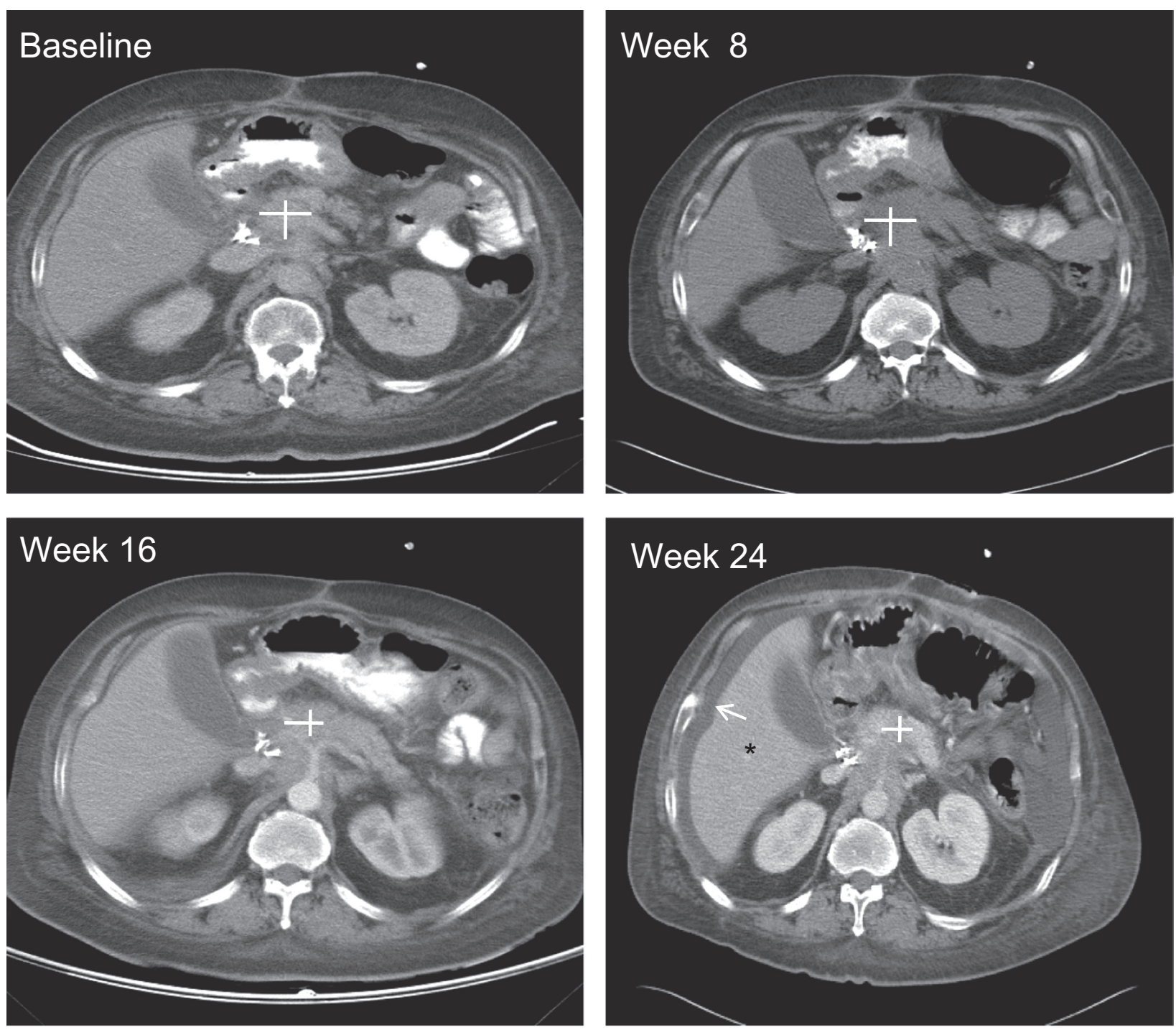

Figure 2 Opioid growth factor (OGF) decreases and/or stabilizes growth of pancreatic cancer. Radiographic images by computerized tomography are shown demonstrating the sections through the primary pancreatic tumor in the head of the pancreas. The pancreatic tumor (marked with a white + ) is shown at baseline and every eight weeks during OGF therapy. The primary tumor size decreased during the study, but at week 24 the patient was noted to have ascites in the peritoneal cavity (arrow) and an II mm nodule in the liver $(*)$ suggestive of progression of disease.

was at least three times that of the hospice control subjects $(p<0.001)$. Increased survival probability of patients treated with OGF compared to hospice patients over time is plotted according to the Kaplan-Meier survival curve (Figure 3). Survival data are shown in Table 3 for both the hospice-referred and the OGF-treated subjects in which the cumulative proportion of those surviving at specific time points are portrayed. At the median survival date of 65 days when $50 \%$ of the OGF-treated subjects are still living, $84 \%$ or 139 subjects in the hospice group have died. Moreover, at the median survival time of 21 days for the hospice patients, none of the OGF-treated subjects had died. Although both groups had a few survivors past
225 days, only $2.4 \%$ of hospice patients fell into this group compared to $17 \%$ of the OGF-treated subjects.

\section{Quality of life surveys}

The mean Karnofsky performance status for the OGF-treated subjects at baseline was $80 \%$ and remained unchanged throughout the duration of the study; no statistical significant change was observed. The overall total scores recorded in the Sickness Impact Profile ${ }^{23}$ between baseline and four weeks $(p=0.49)$ and from baseline to eight weeks $(p=0.90)$. Of the twelve subcategories measured in the Sickness Impact Profile, the categories of "Alertness Behavior" improved from baseline values at week 8 by $22.5 \%$ whereas "Communication" 


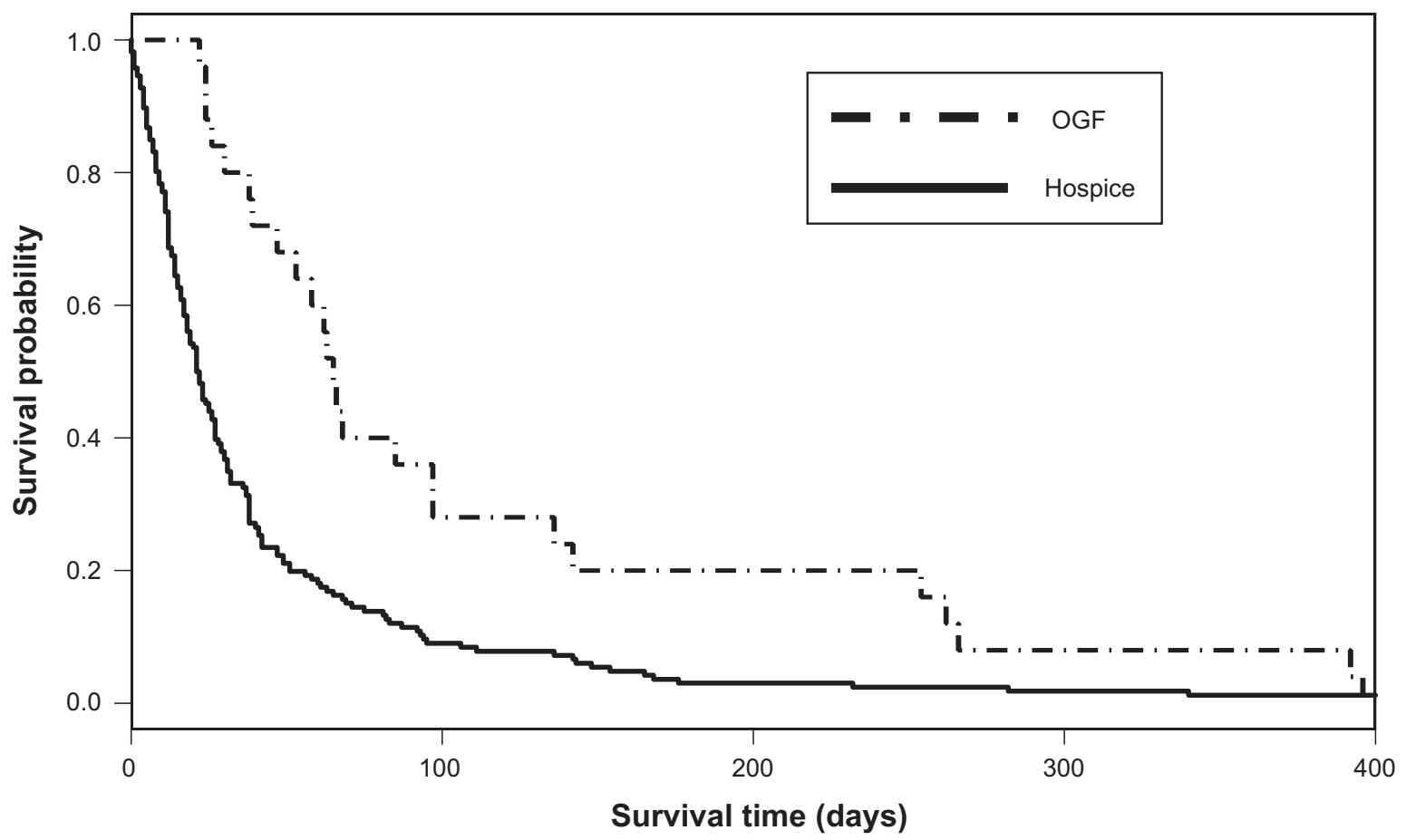

Figure 3 Survival of advanced pancreatic cancer is improved compared to untreated controls. A) Opioid growth factor (OGF)-treated patients survived for 209\% longer than untreated controls. B) Survival of OGF-treated subjects over time compared to control subjects using the Kaplan-Meier curve. The OGF-treated group differed significantly from controls at $p<0.001$.

Notes: Data represent means \pm standard error of mean. ${ }^{* * *}$ Significantly different from hospice controls, $p<0.001$.

improved by $29 \%$ and $36 \%$ at weeks 4 and 8 , respectively; these trends did not meet statistical significance. The subcategory in the Sickness Impact Profile of "Ambulation" decreased at week 8 in the study patients $(p=0.036)$.

There was no change in the Beck Depression survey ${ }^{24}$ at either week $4(p=0.57)$ or week $8(p=0.78)$. There also were no statistically significant differences in pain perception as assessed by the McGill Pain Questionnaire ${ }^{25}$ from baseline to weeks 4 or 8 of the study ( $p=0.377$ and $p=0.98$, respectively).

\section{Laboratory parameters}

There was no change statistically in hematologic, chemistries or hepatic values in OGF-treated individuals from baseline values throughout the study (Table 4). Plasma $\left[\mathrm{Met}^{5}\right]$-enkephalin levels were measured monthly prior to infusion and are shown in Figure 4. [ $\left.\mathrm{Met}^{5}\right]$-enkephalin levels increased from baseline values of $7.89 \pm 2.97 \mathrm{pg} / \mathrm{mL}$ to $19.5 \pm$ $8.7 \mathrm{pg} / \mathrm{mL}$ at week $4(p=0.009)$, and $55.0 \pm 28.8 \mathrm{pg} / \mathrm{mL}$ $(p=0.0001)$ at week 8 .

\section{Toxicities}

There were no severe adverse events related to the infusion of OGF. Table 5 presents the toxicities reported and whether they were considered to be due to the treatment, the primary disease, or concomitant medications. Thirty-two percent of the patients reported abdominal pain and nausea, which may have been due to the OGF infusion or the underlying metastatic cancer. Twenty percent of the subjects reported constipation which may have been related to concomitant narcotics administered for pain control. Three subjects reported bruising and two of these were on warfarin therapy for deep venous thrombosis; hence, the bruising may have been due to the anticoagulant. Two patients died of acute pulmonary embolism at weeks 3 and 7. The other symptoms reported were most likely due to the OGF infusion and were reported as grade 1 toxicities; none of these toxicities required a dose reduction or discontinuation of treatment. There were no hematologic toxicities reported.

\section{Conclusions}

This study reports the results of a phase II clinical trial using a novel biotherapy, OGF, for the treatment of advanced pancreatic cancer. The design of a phase II clinical trial typically includes naïve subjects, such that tumor resistance is not an issue and an untreated group receives placebo. Since OGF had not previously been examined for efficacy in cancer therapy (ie, phase II clinical trials), and the FDA did 
Table 3 Survival tables

\begin{tabular}{|c|c|c|c|c|c|c|}
\hline Stratum & Time & Status & Survival & SEM & Failed & Left \\
\hline hospice & 0 & $I$ & 0.9819 & 0.0103 & 3 & 163 \\
\hline hospice & I & I & 0.9578 & 0.0156 & 7 & 159 \\
\hline hospice & 2 & I & 0.9458 & 0.0176 & 9 & 157 \\
\hline hospice & 3 & I & 0.9277 & 0.0201 & 12 & 154 \\
\hline hospice & 4 & I & 0.8976 & 0.0235 & 17 & 149 \\
\hline hospice & 5 & I & 0.8675 & 0.0263 & 22 & 144 \\
\hline hospice & 6 & I & 0.8494 & 0.0278 & 25 & $14 \mid$ \\
\hline hospice & 7 & I & 0.8313 & 0.0291 & 28 & 138 \\
\hline hospice & 8 & I & 0.8012 & 0.031 & 33 & 133 \\
\hline hospice & 9 & I & 0.7831 & 0.032 & 36 & 130 \\
\hline hospice & 10 & I & 0.7711 & 0.0326 & 38 & 128 \\
\hline hospice & 11 & I & $0.74 I$ & 0.034 & 43 & 123 \\
\hline hospice & 12 & I & 0.6867 & 0.036 & 52 & 114 \\
\hline hospice & 13 & I & 0.6747 & 0.0364 & 54 & 112 \\
\hline hospice & 14 & I & 0.6446 & 0.0371 & 59 & 107 \\
\hline hospice & 15 & I & 0.6265 & 0.0375 & 62 & 104 \\
\hline hospice & 16 & I & 0.6084 & 0.0379 & 65 & 101 \\
\hline hospice & 17 & I & 0.5843 & 0.0383 & 69 & 97 \\
\hline hospice & 18 & I & 0.5602 & 0.0385 & 73 & 93 \\
\hline hospice & 19 & I & 0.5422 & 0.0387 & 76 & 90 \\
\hline hospice & 20 & I & $0.536 \mathrm{I}$ & 0.0387 & 77 & 89 \\
\hline hospice & 21 & I & 0.5 & 0.0388 & 83 & 83 \\
\hline hospice & 22 & I & 0.4819 & 0.0388 & 86 & 80 \\
\hline hospice & 23 & I & 0.4578 & 0.0387 & 90 & 76 \\
\hline hospice & 24 & I & 0.4518 & 0.0386 & 91 & 75 \\
\hline hospice & 25 & I & 0.4398 & 0.0385 & 93 & 73 \\
\hline hospice & 26 & I & 0.4277 & 0.0384 & 95 & 71 \\
\hline hospice & 27 & I & 0.3976 & 0.038 & 100 & 66 \\
\hline hospice & 28 & I & 0.3916 & 0.0379 & 101 & 65 \\
\hline hospice & 29 & I & 0.3795 & 0.0377 & 103 & 63 \\
\hline hospice & 30 & I & 0.3675 & 0.0374 & 105 & 61 \\
\hline hospice & 31 & I & 0.3494 & 0.037 & 108 & 58 \\
\hline hospice & 32 & I & 0.3313 & 0.0365 & 111 & 55 \\
\hline hospice & 36 & I & 0.3253 & 0.0364 & 112 & 54 \\
\hline hospice & 37 & 1 & 0.3133 & 0.036 & 114 & 52 \\
\hline hospice & 38 & I & 0.2711 & 0.0345 & 121 & 45 \\
\hline hospice & 40 & I & 0.2651 & 0.0343 & 122 & 44 \\
\hline hospice & 41 & I & 0.253 & 0.0337 & 124 & 42 \\
\hline hospice & 42 & I & 0.2349 & 0.0329 & 127 & 39 \\
\hline hospice & 47 & I & 0.2229 & 0.0323 & 129 & 37 \\
\hline hospice & 49 & I & 0.2108 & 0.0317 & $13 \mid$ & 35 \\
\hline hospice & 51 & I & 0.1988 & 0.031 & 133 & 33 \\
\hline hospice & 56 & I & 0.1928 & 0.0306 & 134 & 32 \\
\hline hospice & 58 & I & 0.1867 & 0.0302 & 135 & 31 \\
\hline hospice & 60 & I & 0.1807 & 0.0299 & 136 & 30 \\
\hline hospice & 61 & I & 0.1747 & 0.0295 & 137 & 29 \\
\hline hospice & 63 & I & 0.1687 & 0.0291 & 138 & 28 \\
\hline hospice & 65 & I & 0.1627 & 0.0286 & 139 & 27 \\
\hline
\end{tabular}

(Continued)
Table 3 (Continued)

\begin{tabular}{|c|c|c|c|c|c|c|}
\hline Stratum & Time & Status & Survival & SEM & Failed & Left \\
\hline hospice & 68 & $I$ & 0.1566 & 0.0282 & 140 & 26 \\
\hline hospice & 69 & 1 & 0.1506 & 0.0278 & $14 \mid$ & 25 \\
\hline hospice & 71 & 1 & 0.1446 & 0.0273 & 142 & 24 \\
\hline hospice & 75 & 1 & 0.1386 & 0.0268 & 143 & 23 \\
\hline hospice & 81 & 1 & 0.1325 & 0.0263 & 144 & 22 \\
\hline hospice & 82 & 1 & 0.1265 & 0.0258 & 145 & 21 \\
\hline hospice & 83 & 1 & 0.1205 & 0.0253 & 146 & 20 \\
\hline hospice & 87 & 1 & 0.1145 & 0.0247 & 147 & 19 \\
\hline hospice & 92 & 1 & 0.1084 & $0.024 I$ & 148 & 18 \\
\hline hospice & 93 & I & 0.1024 & 0.0235 & 149 & 17 \\
\hline hospice & 94 & I & 0.0964 & 0.0229 & 150 & 16 \\
\hline hospice & 95 & I & 0.0904 & 0.0223 & $15 \mid$ & 15 \\
\hline hospice & 106 & I & 0.0843 & 0.0216 & 152 & 14 \\
\hline hospice & III & I & 0.0783 & 0.0209 & 153 & 13 \\
\hline hospice & 136 & I & 0.0723 & 0.0201 & 154 & 12 \\
\hline hospice & 142 & I & 0.0663 & 0.0193 & 155 & 11 \\
\hline hospice & 143 & I & 0.0602 & 0.0185 & 156 & 10 \\
\hline hospice & 148 & 1 & 0.0542 & 0.0176 & 157 & 9 \\
\hline hospice & 154 & 1 & 0.0482 & 0.0166 & 158 & 8 \\
\hline hospice & 165 & I & 0.0422 & 0.0156 & 159 & 7 \\
\hline hospice & 168 & I & 0.0361 & 0.0145 & 160 & 6 \\
\hline hospice & 176 & I & 0.0301 & 0.0133 & 161 & 5 \\
\hline hospice & 232 & 1 & 0.0241 & 0.0119 & 162 & 4 \\
\hline hospice & 282 & 1 & 0.0181 & 0.0103 & 163 & 3 \\
\hline hospice & 340 & I & 0.012 & 0.0085 & 164 & 2 \\
\hline hospice & 499 & 1 & 0.00602 & 0.006 & 165 & 1 \\
\hline Stratum & Time & Study & Survival & SEM & Failed & Left \\
\hline study & 0 & $\mathrm{I}$ & $I$ & 0 & 0 & 24 \\
\hline study & 22 & 1 & 0.9583 & 0.0408 & 1 & 23 \\
\hline study & 24 & I & 0.875 & 0.0675 & 3 & 21 \\
\hline study & 26 & 1 & 0.8333 & $0.076 \mathrm{I}$ & 4 & 20 \\
\hline study & 30 & I & 0.7917 & 0.0829 & 5 & 19 \\
\hline study & 38 & I & 0.75 & 0.0884 & 6 & 18 \\
\hline study & 39 & I & 0.7083 & 0.0928 & 7 & 17 \\
\hline study & 53 & I & 0.6667 & 0.0962 & 8 & 16 \\
\hline study & 58 & 1 & 0.625 & 0.0988 & 9 & 15 \\
\hline study & 62 & I & 0.5833 & 0.1006 & 10 & 14 \\
\hline study & 63 & 1 & 0.5417 & 0.1017 & 11 & 13 \\
\hline study & 65 & 1 & 0.5 & 0.1021 & 12 & 12 \\
\hline study & 66 & 1 & 0.4583 & 0.1017 & 13 & 11 \\
\hline study & 68 & 1 & 0.4167 & 0.1006 & 14 & 10 \\
\hline study & 85 & I & 0.375 & 0.0988 & 15 & 9 \\
\hline study & 97 & I & 0.2917 & 0.0928 & 17 & 7 \\
\hline study & 136 & 1 & 0.25 & 0.0884 & 18 & 6 \\
\hline study & 142 & I & 0.2083 & 0.0829 & 19 & 5 \\
\hline study & 254 & I & 0.1667 & $0.076 \mathrm{I}$ & 20 & 4 \\
\hline study & 262 & I & 0.125 & 0.0675 & 21 & 3 \\
\hline study & 266 & 1 & 0.0833 & 0.0564 & 22 & 2 \\
\hline study & 392 & 1 & 0.0417 & 0.0408 & 23 & 1 \\
\hline
\end{tabular}


Table 4 Laboratory values

\begin{tabular}{lll}
\hline Laboratory test & Baseline & Week 8 \\
\hline Hemoglobin g/dL & $1 \mathrm{l} .2 \pm 0.3$ & $1 \mathrm{I} .4 \pm 0.4$ \\
White blood cell count $(\mathrm{k} / \mu \mathrm{L})$ & $7.3 \pm 0.7$ & $7.6 \pm 0.6$ \\
Absolute neutrophils $(\mathrm{k} / \mu \mathrm{L})$ & $5.6 \pm 0.7$ & $5.7 \pm 0.7$ \\
Platelet count $(\mathrm{k} / \mu \mathrm{L})$ & $196 \pm 20$ & $172 \pm 19$ \\
Alkaline phosphatase $(\mathrm{U} / \mathrm{L})$ & $189 \pm 3 \mathrm{I}$ & $344 \pm 140$ \\
ALT $(\mathrm{U} / \mathrm{L})$ & $25.8 \pm 3.4$ & $30.9 \pm 8.4$ \\
AST $(\mathrm{U} / \mathrm{L})$ & $33.5 \pm 3.7$ & $35.2 \pm 7.8$ \\
Bilirubin, total $(\mathrm{mg} / \mathrm{dL})$ & $0.8 \pm 0.2$ & $1.0 \pm 0.3$ \\
Albumin $(\mathrm{g} / \mathrm{dL})$ & $3.5 \pm 0.1$ & $3.1 \pm 0.2$ \\
BUN $(\mathrm{mg} / \mathrm{dL})$ & $13.8 \pm 1.4$ & $15.6 \pm 2.0$ \\
Creatinine $(\mathrm{mg} / \mathrm{dL})$ & $0.8 \pm 0.1$ & $0.8 \pm 0.1$ \\
Glucose $(\mathrm{mg} / \mathrm{dL})$ & $115 \pm 1 \mathrm{I}$ & $129 \pm 14$ \\
Calcium $(\mathrm{mg} / \mathrm{dL})$ & $12.6 \pm 4.0$ & $8.4 \pm 0.2$ \\
Sodium $(\mathrm{mmol} / \mathrm{L})$ & $137.3 \pm 0.7$ & $135.5 \pm$ \\
Potassium $(\mathrm{mmol} / \mathrm{L})$ & & 1.6 \\
INR & $3.9 \pm 0.1$ & $4.0 \pm 0.1$ \\
\hline
\end{tabular}

Notes: Values represent the mean \pm SEM at baseline $(N=25)$ and at week-8 $(N=13)$. Abbreviations: ALT, alanine aminotransferase; AST, aspartate aminotransferase; BUN, blood urea nitrogen; INR, international normalized ratio.

not allow naïve subjects to be treated with this medication, therefore, only individuals who had already failed chemotherapy were eligible for study. For ethical reasons, a placebo control group is not employed when treating cancer patients and an IRB does not typically grant permission for this type of study design. Thus, a large control population of patients in this investigation was established from subjects meeting the same inclusion criteria but who desired no further care after failing chemotherapy. One limitation of this study was that it was not a randomized controlled study; therefore, a risk of selection bias may have occurred in that those selecting hospice care may have had a poorer performance status. Unfortunately, the dismal prognosis of pancreatic cancer is only counted in months rather than years and survival with standard chemotherapy using gemcitabine is only reported only for 5.6 months. ${ }^{30}$ Treatment with any agent at this end-stage of a disease may seem fruitless, whereas others would support that any agent showing improvement with advanced cancer may have potential if used earlier or shortly after diagnosis.

Since chemotherapy has not been very beneficial for survival in the treatment of pancreatic cancer, investigators are studying alternative benefits of treating subjects with advanced disease..$^{4,10,30}$ The current investigation demonstrated a clinical benefit for improvement in pain, functional status, and weight. Additionally, survival was also improved in those subjects treated with OGF even at this late stage. The historical controls to which we compared our OGF-treated patients in this study were treatment-naïve upon initiation of the trial rather than treatment failures as in our current study. Even though our patient population had much more advanced disease, the clinical benefit we observed was greater. One reason for this difference may be related to OGF being a natural endogenous peptide so off-target toxicity was not as pronounced as that with chemotherapy.

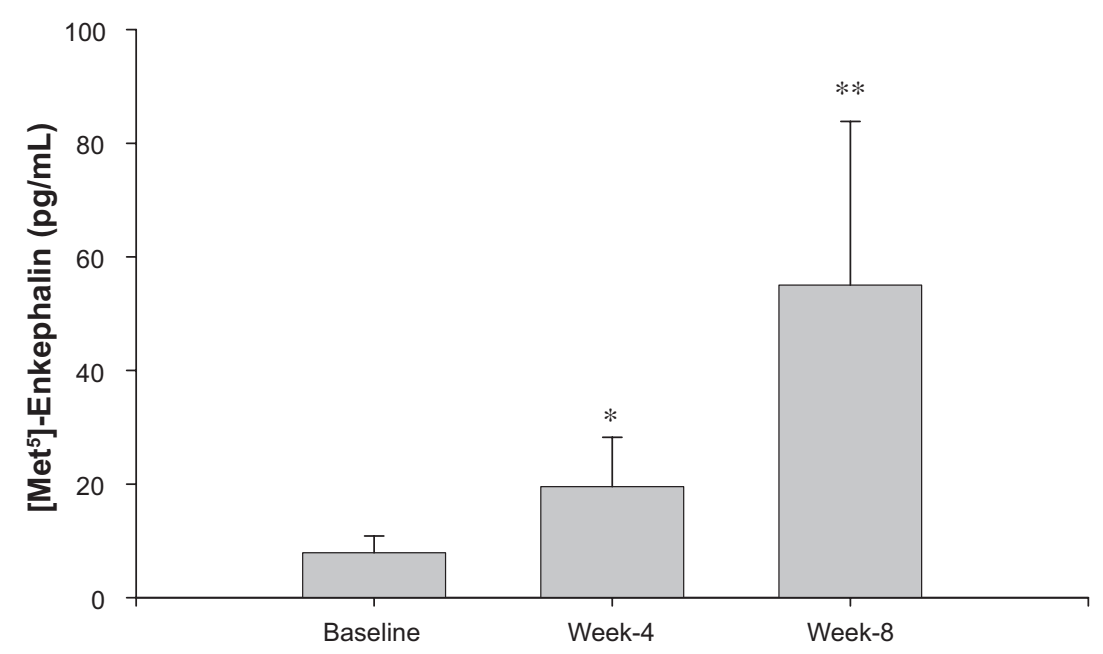

Figure 4 Plasma $\left[\right.$ Met $\left.^{5}\right]$-enkephalin levels in opioid growth factor (OGF)-treated patients over time. The antibody used for this assay was highly specific for [Met $\left.{ }^{5}\right]$-enkephalin with little or no cross reactivity with B-endorphin, dynorphin A, ACTH and endothelin-I.The assay range was $10-1280 \mathrm{pg} / \mathrm{mL}$. Between run precision at concentrations of 21 and $636 \mathrm{pg} / \mathrm{mL}$ averaged $16 \%$ and II\% CV, respectively, and the lower limit of quantitation was $8 \mathrm{pg} / \mathrm{mL}$.

Notes: Data are presented as means \pm standard error of mean for baseline, week 4 , and week 8 for subjects treated with OGF. Significantly different from baseline are represented by $* p<0.01$, and $* * p<0.001$. 
Table 5 Toxicities

\begin{tabular}{lll}
\hline Toxicity & No & $\%$ \\
\hline Nausea & $8^{*}$ & $32^{*}$ \\
Abdominal pain & 8 & $32^{*}$ \\
Paresthesias & 6 & 24 \\
Constipation & 5 & $20^{*}$ \\
Dry mouth & 4 & 16 \\
Lightheadedness & 4 & 16 \\
Flushing & 3 & 12 \\
Diarrhea & 3 & 12 \\
Bruising & 3 & $12 *$ \\
Hypotension & 2 & 8 \\
Confusion & 2 & 8 \\
Red tongue & 2 & 8 \\
Hiccoughs & 1 & 4 \\
Euphoria & 1 & 4 \\
Dry eyes & 1 & 4 \\
Blurry vision & 1 & 4 \\
\hline
\end{tabular}

Notes: *May have been due to underlying disease or concomitant medications rather than OGF.

In comparison to subjects electing no further treatment, OGF-treated patients survived three times longer. In fact, more than half of the cancer patients treated with OGF had either regression of tumor size or stabilization of disease. These results extend the findings in a prior study where OGF was used in an acute dose finding protocol. ${ }^{21}$ In the same study, when subjects were treated with extended therapy, a mean survival of 8.7 months (261 days) in the intravenoustreated group and 9.5 months (291 days) in OGF-treated group via a subcutaneous route was reported. ${ }^{21}$ Survival in this previous study was 2.4 times greater than that in the current study, and $60 \%$ greater than for historical controls treated with gemcitabine. ${ }^{30}$ The possible rationale for the extended survival advantage in the prior study may be because $56 \%$ of the subjects were treatment-naïve. This finding provokes the need to study OGF in previously untreated pancreatic cancer patients.

Three quality of life surveys were conducted during the course of this study. Although none of the surveys showed significant improvements with OGF, they also did not show that the quality of life deteriorated. Crippa and colleagues ${ }^{31}$ recently published results of a quality of life analysis in different stages of pancreatic cancer. Using the Functional Assessment of Cancer Therapy survey, in contrast to the three quality of life surveys employed in the current study, Crippa and colleagues concluded that the quality of life significantly decreased in subjects with metastatic pancreatic cancer. These investigators thought that the decreased quality of life was either due to the chemotherapy or the progression of the disease. Since biotherapy with OGF did not cause the same toxic side effects as chemotherapy, this may account for the fact that patients in the present study did not show a decrease in their quality of life. Another reason that OGF treatment did not diminish quality of life may be related to the fact that the progression of disease was slowed and survival increased.

The well-known toxicities reported with standard chemotherapeutic agents used in the treatment of pancreatic cancer, such as hematologic toxicities from bone marrow suppression $^{32}$ and gastrointestinal mucosynovitis, ${ }^{33}$ was not observed with OGF. Other toxicities like hand-and-foot syndrome (palmar-plantar erythrodysesthesia or chemotherapyinduced acral erythema) ${ }^{34}$ seen with capecitabine therapy for pancreatic cancer, were similarly not observed in our study. Hypotension has been reported previously in pancreatic cancer patients treated with OGF in a phase I clinical trial ${ }^{21}$ with an occurrence of $43 \%$. However, the incidence of hypotension in individuals enrolled this current study was less at $8 \%$. This decreased incidence of hypotension was most likely due to the prolongation of the infusion time from 30 to 45 minutes utilized in the present study. Two subjects died prematurely from pulmonary embolism in this phase II trial. It is known that many patients with pancreatic cancer are in a hypercoagulable state (Trousseau's syndrome), increasing the potential and incidence of thromboembolic events (both venous and arterial), particularly in the setting of advanced disease. ${ }^{35}$ Therefore, the occurrence of thrombophlebitis and pulmonary embolism in our two subjects may have been due to the pancreatic cancer rather than the treatment with OGF. However, Stein and colleagues ${ }^{36}$ found only $0.16 \%$ of deaths in pancreatic cancer subjects were related to pulmonary emboli; hence, further investigation is needed to determine whether this complication is a coincidence or potential side effect.

Plasma enkephalin levels increased over the course of this study which may have been attributed to the administration of intravenous [Met $\left.{ }^{5}\right]$-enkephalin (OGF) and its accumulation in tissues. We previously reported that plasma enkephalin values were increased in human subjects with advanced pancreatic cancer without OGF treatment. ${ }^{37}$ The reason for the increase in the current study may be due to several possibilities including: tissue accumulation, a compromising of the OGF-OGFr pathway, or the body's natural response to fighting off a malignancy.

Since clinical benefit and survival in our patients with advanced pancreatic cancer who had previously failed other 
treatments was markedly improved, these data support the rationale for testing this biological agent in the treatment of naïve patients where the tumor burden is less and perhaps not yet drug resistant. Additionally, further studies whereby OGF is combined with standard therapy such as gemcitabine to potentiate the cytotoxic effects of chemotherapy drugs should also be considered. Indeed, we have shown in vitro and in an animal model that the combination of gemcitabine with OGF significantly decreases pancreatic cancer tumor growth compared to either agent alone, and OGF treatment also reduced the toxic effects of gemcitabine. ${ }^{38}$ Given the aggressive nature of pancreatic cancer and its resistance to standard chemotherapeutic regimens, it may be that OGF in combination with gemcitabine given at the time of diagnosis may be a powerful therapeutic modality for treatment of this deadly disease.

\section{Acknowledgments}

We thank Karen Paris and Hospice of Central Pennsylvania for their participation in providing information about the control subjects for this study. We also thank the nursing staff of the General Clinical Research Center (GCRC) from the Pennsylvania State University.

\section{Disclosures}

Pennsylvania State University holds a patent for the use of OGF in pancreatic cancer and other malignancies. Drs. Smith and Zagon are co-inventors. This work was supported by a grant from the Food and Drug Administration Orphan Drug [Grant \#002391] and National Institutes of Health [Grant numbers M01-RR10732, C06 RR016499].

\section{References}

1. Rocha Lima CM, Centeno B. Update on pancreatic cancer. Curr Opin Oncol. 2002;14(4):424-430.

2. Jemal A, Siegel R, Ward E, et al. Cancer statistics, 2008. CA Cancer J Clin. 2008;58(2):71-96.

3. Bramhall S, Dunn JA, Neoptolemos JP. Epidemiology of pancreatic cancer. In: Beger HG, Warshaw AL, Buchler MW, et al. editors. The Pancreas. Cambridge, MA: Blackwell Science, Inc., 1998.

4. Goldstein D, Carroll S, Apte M, et al. Modern management of pancreatic carcinoma. Intern Med J. 2004;34(8):475-481.

5. Heinemann V. Gemcitabine-based combination treatment of pancreatic cancer. Semin Oncol. 2002;29(1 Supp1 3):25-35.

6. McGinn CJ, Lawrence TS, Zalupski MM. On the development of gemcitabine-based chemoradiotherapy regimens in pancreatic cancer. Cancer. 2002;95(4 Suppl):933-940.

7. Oettle H, Riess H. Gemcitabine in combination with 5-fluorouracil with or without folinic acid in the treatment of pancreatic cancer. Cancer. 2002;95(4 Suppl):912-922.

8. Blackstock AW, Bernard SA, Richards F, et al. Phase I trial of twice-weekly gemcitabine and concurrent radiation in patients with advanced pancreatic cancer. J Clin Oncol. 1999;17(7): 2208-2212.
9. Wolff RA. Novel therapies for pancreatic cancer. Cancer $J$. 2001;7(4):349-358.

10. Burris HA III, Moore MJ, Andersen J, et al. Improvements in survival and clinical benefit with gemcitabine as first-line therapy for patients with advanced pancreas cancer: a randomized trial. J Clin Oncol. 1997;15(6):2403-2413.

11. Ozawa F, Friess H, Tempia-Caliera A, et al. Growth factors and their receptors in pancreatic cancer. Teratog Carcinog Mutagen. 2001;21(1):27-44.

12. Reddy SA. Signaling pathways in pancreatic cancer. Cancer J. 2001;7(4):274-286.

13. Shi X, Friess H, Kleeff J, et al. Pancreatic cancer: factors regulating tumor development, maintenance and metastasis. Pancreatology. 2001;1(5):517-524.

14. Zagon IS, Verderame MF, McLaughlin PJ. The biology of the opioid growth factor receptor (OGFr). Brain Res Brain Res Rev. 2002;38(3):351-376.

15. Zagon IS, Smith JP, McLaughlin PJ. Human pancreatic cancer cell proliferation in tissue culture is tonically inhibited by opioid growth factor. Int J Oncol. 1999;14(3):577-584.

16. Zagon IS, Smith JP, Conter R, et al. Identification and characterization of opioid growth factor receptor in human pancreatic adenocarcinoma. Int J Mol Med. 2000;5(1):77-84.

17. Zagon IS, Hytrek SD, Smith JP, et al. Opioid growth factor (OGF) inhibits human pancreatic cancer transplanted into nude mice. Cancer Lett. 1997;112(2):167-175.

18. Zagon IS, Roesener CD, Verderame MF, et al. Opioid growth factor regulates the cell cycle of human neoplasias. Int J Oncol. 2000;17(5): 1053-1061.

19. Zagon IS, Ruth TB, Leure-duPree AE, et al. Immunoelectron microscopic localization of the opioid growth factor receptor (OGFr) and OGF in the cornea. Brain Res. 2003;967(1-2):37-47.

20. Zagon IS, Verderame MF, Allen SS, et al. Cloning, sequencing, chromosomal location, and function of cDNAs encoding an opioid growth factor receptor (OGFr) in humans. Brain Res. 2000;856(1-2):75-83.

21. Smith JP, Conter RL, Bingaman SI, et al. Treatment of advanced pancreatic cancer with opioid growth factor: phase I. Anticancer Drugs. 2004;15(3):203-209.

22. Karnofsky DA, Abelmann WH, Craver LF, et al. Performance status in cancer patients. Cancer. 1948;1:634-656.

23. Bergner M. Development, testing, and the use of the Sickness Impact Profile. In: Walker SR, Rosser RM, editors. Quality of Life: Assessment and Application. Boston, MA: MTP Press Limited, 1987.

24. Beck AT, Ward CH, Medelson M, et al. An inventory for measuring depression. Arch Gen Psychiatry. 1961;4:561-571.

25. Melzack R. The McGill Pain Questionnaire: major properties and scoring methods. Pain. 1975;1(3):277-299.

26. National Cancer Institute. Cancer Therapy Evaluation Program: Common toxicity criteria. Version 2.0 DCTD. 3-23-1998. Bethesda, MD: National Cancer Institute, National Institutes of Health; 1998.

27. Therasse P, Arbuck SG, Eisenhauer EA, et al. New guidelines to evaluate the response to treatment in solid tumors. European Organization for Research and Treatment of Cancer, National Cancer Institute of the United States, National Cancer Institute of Canada. J Natl Cancer Inst. 2000;92(3):205-216.

28. National Cancer Institute. Common Terminology Criteria for Adverse Events v3.0 (CTCAE). 8-9-2006. Bethesda, MD: National Cancer Institute, National Institutes of Health; 2006.

29. Kaplan EL, Meier P. Nonparametric estimation from incomplete observations. J Am Stat Assoc. 1958;53:457-481.

30. Burris H, Storniolo AM. Assessing clinical benefit in the treatment of pancreas cancer: gemcitabine compared to 5-fluorouracil. Eur J Cancer. 1997;33(Suppl 1):S18-S22.

31. Crippa S, Dominguez I, Rodriguez JR, et al. Quality of life in pancreatic cancer: analysis by stage and treatment. $J$ Gastrointest Surg. 2008;12(5):783-793.

32. Green MR. Gemcitabine safety overview. Semin Oncol. 1996;23(5 Suppl 10):32-35. 
33. Sonis ST. Mucositis as a biological process: a new hypothesis for the development of chemotherapy-induced stomatotoxicity. Oral Oncol. 1998;34(1):39-43.

34. Cartwright TH, Cohn A, Varkey JA, et al. Phase II study of oral capecitabine in patients with advanced or metastatic pancreatic cancer. J Clin Oncol. 2002;20(1):160-164.

35. Sack GH Jr, Levin J, Bell WR. Trousseau's syndrome and other manifestations of chronic disseminated coagulopathy in patients with neoplasms: clinical, pathophysiologic, and therapeutic features. Medicine (Baltimore). 1977;56(1):1-37.
36. Stein PD, Beemath A, Meyers FA, et al. Pulmonary embolism as a cause of death in patients who died with cancer. Am J Med. 2006;119(2): $163-165$.

37. Smith JP, Conter RL, Demers LM, et al. Elevated levels of opioid growth factor in the plasma of patients with pancreatic cancer. Pancreas. 2000;21(2):158-164.

38. Zagon IS, Jaglowski JR, Verderame MF, et al. Combination chemotherapy with gemcitabine and biotherapy with opioid growth factor (OGF) enhances the growth inhibition of pancreatic adenocarcinoma. Cancer Chemother Pharmacol. 2005;56(5):510-520.

\section{Publish your work in this journal}

The Open Access Journal of Clinical Trials is an international, peerreviewed, open access journal publishing original research, reports, editorials, reviews and commentaries on all aspects of clinical trial design, management, legal, ethical and regulatory issues, case record form design, data collection, quality assurance and data auditing methodologies. The manuscript management system is completely online and includes a very quick and fair peer-review system, which is all easy to use. Visit http://www.dovepress.com/testimonials.php to read real quotes from published authors.

Submit your manuscript here: http://www.dovepress.com/open-access-journal-of-clinical-trials-journal 